\title{
SER Y ACCIÓN SOCIAL EN EL HORIZONTE DE LA REFLEXIÓN ACERCA DEL TIEMPO DESARROLLADA POR HEIDEGGER EN SER Y TIEMPO
}

Being and social action in the horizon of Heidegger's thinking on temporality in Being and Time

Domingo Fernández Agis*

Resumen

Según plantea Heidegger en Ser y tiempo, la pregunta por el ser no es fruto de la espontaneidad de la llamada conciencia natural, aunque tampoco en la racionalidad científica pueda encontrar los elementos adecuados para su formulación. Sabemos, en todo caso, que la ciencia puede contribuir a la respuesta, aunque no ayude a comprender lo que el hecho mismo de esa enunciación implica. La tesis que se defiende en este trabajo es que la correlación entre la pregunta por el ser y la pregunta por el tiempo, posee unas dimensiones objetiva, subjetiva e histórica, estas tres dimensiones todavía no han sido, a mi entender, suficientemente exploradas. La conclusión a que se llega puede resumirse afirmando que en cada una de ellas acaba implicado el problema de la autenticidad, un problema que no puede ser tratado de forma adecuada desde la concepción heideggeriana del tiempo desarrollada en Ser y tiempo.

Palabras clave: Heidegger, Acción, Sociedad, Tiempo, Autenticidad.

Abstract

According to what Heidegger exposed in Being and Time, the question of being is not the result of the spontaneity of what we call natural consciousness, although we cannot find in scientific rationality the right items for its formulation. We know, in any case, that science can contribute to the answer, but does not help us understand what the fact of this statement implies. The thesis defended in this work is that the correlation between the question of being and the question of the time has an objective, subjective and historical dimension, not yet fully explored. The conclusion reached is that in all of them is involved the problem of authenticity, that can't be addressed adequately from Heidegger's conception of time exposed in Being and Time.

Key words: Heidegger, Action, Society, Time, Authenticity.

\section{INTRODUCCIÓN}

Siguiendo cierta estela característica de quienes de diverso modo han administrado la herencia hegeliana, podría decirse que, porque el ser es tiempo, se despliega en el devenir y se hace historia. A esto podría añadirse, intentando con ello eludir el riesgo de perdernos en la infinita capilaridad de la existencia, que la historia del ser solo puede ser narrada relatando el devenir del pensamiento. Como decía, se advierte sin ninguna dificultad el carácter hegeliano de esta apreciación. Me gustaría 
ahora realizar una aproximación a este asunto, partiendo de algunos parágrafos de Ser y tiempo.

Hay que decir ante todo, que pese a esa aparente sintonía entre planteamientos que a priori no podrían ser más dispares, existen elementos significativos que vienen a subrayar las diferencias entre ambas filosofias. Hay ante todo, un signo diferencial que ha de destacarse de antemano. Me refiero a la ausencia del momento final de la reconciliación que existe, no ya en el método dialéctico, sino en los incontables terrenos de aplicación que encuentran para él quienes lo consideran la mejor de las metodologías posibles. En efecto, no encontramos en esta obra horizonte de salida que posibilite remontar la negatividad de lo real, como menos aún la hay para la Historia, pues ni siquiera esta última puede pensarse como continuidad o unidad final en el tiempo. No impide esto considerar que el pensar es histórico, por más que articular esa historicidad en discurso sea una tarea no exenta de dificultades. Provienen estas, en la mayor parte de los casos, de una filosofía que en su mismo punto de arranque ha sentado como presupuesto la imposibilidad del ser de pensarse a sí mismo. Desde esta perspectiva, que sepamos, tan solo la racionalidad humana podría pensar el ser, pero, aun así, cabe preguntarse si podrá remontar su circunstancia hasta una posición en la que sea posible dominar un horizonte no limitado por cuanto le obliga a atenerse a su posición existencial. En mi opinión, pese a la complejidad del lenguaje utilizado en Ser y tiempo, Heidegger ha dejado claro en esta obra que esa tarea desborda la existencia del ser-ahí, que es quien está en mejor disposición para pensar el ser. Pero eso no significa que haya que renunciar a trazar ciertas líneas, necesarias, por otra parte, para ganar perspectiva (Krockow 204).

\section{LA PERSPECTIVA FOUCAULTIANA}

Es posible establecer, en este punto, una determinada analogía entre su posición y la de Michel Foucault, ya que, al igual que este, tampoco Heidegger "hace una historia de los sujetos, sino de los procesos de subjetivación, bajo los plegamientos que se efectúan tanto en un campo ontológico como social" (Deleuze, Foucault, 151). A su manera, utilizando un discurso ontológico que no puede, sin embargo, eludir sus continuas proyecciones hacia el ámbito de la ética, el filósofo alemán acaba contraponiendo los resultados de la aplicación de dos modos de subjetivación: el que conduce a la subjetividad indiferenciada de la masa y el que aboca en el modo de ser del Dasein. Puede apreciarse, en este sentido, otro punto de coincidencia entre ambos pensadores pues, como señala Gilles Deleuze, si algo obsesiona a Michel Foucault es comprender la realidad del pensamiento, llegar a determinar con claridad cuál es su significado, qué entendemos por pensar. Así retoma el pensador francés la pregunta planteada por Heidegger, tratando de elaborar "una historia, pero del pensamiento como tal. Pensar es experimentar, es problematizar. El saber, el poder y el sí mismo son la triple raíz de una problematización del pensamiento" (151). 
Tanto por lo que se refiere a la última cuestión, cuanto por lo que dice relación a las otras, las dificultades con las que se encuentran los dos pensadores son de orden mayor (Babich, 20 y ss.). En definitiva, se trata, no podemos olvidarlo, de establecer las posibilidades de construir una subjetividad que se yerga como diferencia y, junto a ella o más bien como mejor expresión de la misma, se trataría asimismo de asentar sobre esa nueva base la virtualidad de un pensar desde la alteridad (Sabot 26). Partiendo de estas premisas, se entiende la complejidad que puede tener pensar el devenir o, dicho sea en expresión clásica, construir el discurso histórico. Puede entenderse asimismo, que sea la propia historicidad (Geschichtlichkeit) del Dasein el horizonte trascendental de dicho proyecto.

\section{PENSANDO EL DEVENIR}

Como muestra de estas dificultades, Heidegger habla de la historia de la ciencia, un campo menos sujeto a la indeterminación que otros que pueden ser objeto de atención dentro de los contenidos de la cultura. Para él, tomando como base el desarrollo de la física, habría que reparar en que su éxito se ha debido a la determinación del carácter matemático o "matematizable" que tienen ciertos procesos naturales. De esa forma, el proyecto de desarrollo de la física "descubre de antemano algo que constantemente está-ahí (la materia) y abre el horizonte para una mirada conductora que considera los momentos constitutivos cuantitativamente determinables de eso que está-ahí (movimiento, fuerza, lugar y tiempo)" (Heidegger, Ser y tiempo, 378).

Podríamos recordar a este respecto la convicción de Galileo Galilei, quien consideraba que la Naturaleza es un libro que está escrito en caracteres matemáticos. De ahí que el lenguaje matemático fuese en su opinión el único adecuado para interpretar los fenómenos naturales. No olvidemos, por otra parte, que Galileo puede ser considerado con todo merecimiento como el fundador de la ciencia moderna. En efecto, al margen de sus importantísimos logros en ciencias como la matemática, la física o la astronomía, el establecimiento que hizo de las bases metodológicas de las ciencias experimentales sería mérito suficiente para hacer de él uno de los científicos más importantes de todos los tiempos.

Las cosas se complican cuando sobrepasamos el ámbito restringido de la historia de la ciencia. Ciertamente, este es un ámbito privilegiado, al fin y al cabo, pues en él es más fácil establecer líneas de continuidad. Es así como basándose en la vigencia de las teorías y a los avatares de su aplicación, es posible reconstruir la parte sustancial de la historia de una ciencia dada. Pero, volviendo a nuestras consideraciones iniciales, habría que profundizar en el condicionamiento de los resultados de la tarea de historiar, en función de las peculiaridades del sujeto que acomete dicha actividad. Es lo que apunta Heidegger al señalar que "si el 'sujeto' se concibe ontológicamente como un Dasein que existe y cuyo ser se funda en la temporalidad, será necesario decir que el mundo es 'subjetivo'. Pero entonces ese 
mundo 'subjetivo', en cuanto tempóreamente trascendente, es 'más objetivo' que todo posible 'objeto"' (Heidegger, Ser y tiempo, 382).

Desde luego, hay que admitir que es así, si ponemos al sujeto heideggeriano en relación con cualquier objeto considerado. No obstante, el problema no sería la relación del ente que denominamos Dasein con cualquier otro ente particular, sino más bien la relación entre aquel y el ser (Tirvaudey, 342). En este sentido, la historia del ser ¿tendría un insalvable carácter subjetivo al estar interferida por la historia del sujeto que lo piensa? No nos pesaría aceptarlo si asumimos previamente la imposibilidad de burlar de una vez para siempre la diferencia ontológica entre ser y ente, así como la intransitabilidad de todo sendero que pretenda conducirnos al reino de la totalidad. Porque la mirada del Dasein no recoge la totalidad de las perspectivas ni se produce desde el interior del Aleph borgiano, hipotético punto desde el que era posible la contemplación perfecta del mundo.

\section{OBJETIVIDAD Y PERSPECTIVA}

En todo caso, tal como se ha venido indicando desde diferentes ángulos, los avatares del concepto de objetividad tampoco resultan fáciles de establecer. A este respecto, Gadamer ha señalado que Heidegger consigue alcanzar en Ser y tiempo una posición desde la que es posible pensar, tanto las similitudes como las diferencias entre el modo griego de entender el conocimiento del mundo y la ciencia moderna. De esa forma, "en el horizonte de su interpretación temporal del ser, la metafísica clásica se le aparece en su conjunto como una ontología de lo que está dado, y la ciencia moderna le parece su heredero inconsciente" (Gadamer, 546). No obstante, no olvida poner de relieve que Heidegger ha advertido la existencia, en el pensamiento griego, de un trasfondo aún no pensado desde la perspectiva de la ciencia moderna.

En esa línea, Gadamer quiere poner en evidencia la dificultad del conocimiento objetivo, pues no nos referimos al usar este término lisa y llanamente al conocimiento del objeto, como tampoco podemos admitir que la objetividad de nuestro método se acopla sin problemas a la realidad dada. Por eso señala que siempre nos "convendrá mantener, tanto la dignidad de la cosa como la objetividad del lenguaje, separadas del prejuicio contra la ontología de lo dado y por lo tanto del concepto de la objetividad" (546).

En cierta forma, el mundo, que se presenta y reconoce como el tener-unmundo de alguien, puede ser considerado subjetivo porque es siempre para un sujeto (Foucault, Mal faire, dire vrai, 162). Esto, huelga decirlo, es mucho más que una cuestión epistemológica, posee una radicalidad existencial que no puede dejarse de lado como si fuese una cuestión marginal. No obstante, cabe decir que la totalidad, como objetivación del conjunto de las perspectivas, es un fruto especulativo de una adición imaginaria, mucho más abstracta que cada una de las perspectivas afines a lo concreto. Por otro lado, no pasa de ser una ilusión, en el mejor de los casos un ideal 
regulativo que proporciona al historiador la esperanzadora visión del final de una tarea que en realidad no puede conocer acabamiento.

Como muestra de ese trascender lo meramente epistémico baste mencionar la forma en que Heidegger introduce un nuevo elemento en este discurso, al apuntar la posibilidad de establecer fines colectivos, mediante el compromiso vital con un objetivo común. Nos dice así, que "sólo en el compartir y en la lucha queda libre el poder del destino común" (Heidegger, Ser y tiempo, 400). Aquí el trasfondo de la objetividad sería un correlato de la reconstrucción de una voluntad intersubjetiva, latente tras el conflicto real de voluntades (Foucault, Soggettività e verità, 40). Aunque bien sabemos que la historia se ha encargado de describir, en una innumerable sucesión de páginas que aún rezuman sangre, las derivas totalitarias de este tipo de discursos. Pero contemplemos algún aspecto más de la cuestión, pues asimismo cabe otro tipo de interpretaciones (Di Giovanni, 55 y ss.).

\section{CONSTRUCCIÓN COLECTIVA Y SUBJETIVIDAD}

En este sentido puede reconocerse como obvio que, sin compartir en alguna medida esfuerzos e inquietudes, no es posible la construcción de ningún futuro colectivo. El mero transcurrir del tiempo, por sí mismo, poco tiene que ver con la construcción de un futuro común. Lo encontrado en un momento histórico concreto, en tanto que fruto azaroso del coestar de los sucesos, no es común en otro sentido que el de poder ser objeto comúnmente percibido. Por otro lado, la cadencia de los lapsos temporales puede acelerarse o enlentecer sin que ello suponga que se va hacia alguna finalidad determinada. Se añade a esto que, el compromiso colectivo con el futuro, se pone en extremo de manifiesto cuando ha de lucharse para defender la posibilidad de ese futuro. Hay aquí una relación de inclusión y exclusión, con una complejidad que no puede ser ignorada. Ante todo habría que determinar cómo se establecen los límites del colectivo y la legitimidad de sus esfuerzos. Sea cual sea la respuesta que les hallemos, sabemos que son estas cuestiones políticas, en la más rancia acepción que pueda tener esa palabra. Recordemos, a este respecto, las palabras de Plutarco, cuando afirma que el ejercicio de la política no se limita tan solo al desempeño de los cargos públicos y, en general, al ejercicio del poder. De la misma forma, señala, que la tarea del filósofo no es repetir lo escrito en los libros. Por el contrario, para él "existe un tipo de política y de filosofia en las que nadie repara" (Plutarco, 50-51), que supone la profunda afinidad entre ambas y una constante acción creativa.

Pero destaquemos, en todo caso, que el compromiso con el destino común es el precio a pagar para que adquiera sentido hablar de una historicidad que puede ser calificada de propia o impropia, en función de la presencia o ausencia de tal compromiso. Bien sabemos que se está deslizando aquí Heidegger por un terreno peligroso. En realidad, estos planteamientos merman la radicalidad de su conceptualización del Dasein, minimizan su determinación ontológica, al admitir la débil posibilidad que existe de que el sujeto de la historia adquiera entidad como 
sujeto colectivo (Foucault, Soggettività e verità, 37-38). En todo caso, para él, frente a la historicidad propia, es posible definir una historicidad impropia. En esta última, "la extensión originaria del destino queda oculta. El Dasein presenta su 'hoy' en la inestabilidad del uno-mismo. Mientras está a la espera de la próxima novedad, ya ha olvidado lo antiguo". Indicando a continuación que, en esta forma impropia de historicidad, "el uno rehuye la elección. Ciego para las posibilidades, es incapaz de repetir lo que ha sido, y se limita a retener y mantener lo 'real' que ha quedado de lo mundanamente histórico ya sido, los restos e informaciones presentes acerca de ello" (Heidegger, Ser y tiempo, 407).

Vemos, por tanto, como la construcción de las posibilidades de la historicidad se hace recaer de nuevo en el Dasein, pese a que Heidegger abre aquí el camino a un acometimiento común de la tarea de decidir. En realidad, no hay otro camino practicable, si no se quiere reescribir en otra clave todo el contenido de Ser y tiempo. Por ello, Heidegger vuelve a la idea inicial de un Dasein que, de alguna manera, carga con el peso de un pasado que no puede asumir ni superar, frente a él ha de definir su estar en el mundo como forma particular de atenerse al presente. En este sentido, piensa que "la existencia impropiamente histórica, cargada con la herencia del 'pasado', irreconocible ya para ella misma, busca, en cambio, lo moderno". Frente a ello, no olvida recalcar que "la historicidad propia comprende la historia como el 'retorno' de lo posible y sabe, por eso, que la posibilidad solo retorna cuando la existencia está destinal-instantáneamente abierta para ella en la repetición resuelta" (Heidegger, Ser y tiempo, 407).

\section{EL “ESPÍRITU” DE LOS TIEMPOS}

Otro aspecto resulta ahora revelador. Se refiere a la ausencia del concepto de espiritu, que de forma casi automática acude a nuestra mente cuando planteamos la historicidad en su clásico sentido de realización o concreción de una realidad no material, que trasciende lo delimitable como específico del individuo. Jacques Derrida ha llamado la atención sobre la desaparición en Ser y tiempo del término espíritu, que es objeto de una cuidadosa evitación por parte de Heidegger. De igual modo, ha indicado cómo el término reaparece con posterioridad a esa obra enmarcado entre comillas, para volver sintomáticamente a hacer su aparición en plenitud en el Discurso del Rectorado (Quesada, 248). Así pues, seis años más tarde, en 1933, Heidegger escribe el Discurso del Rectorado. Con este texto se produce la puesta en escena del concepto de espíritu sin las significativas comillas que antes lo habían apostillado. "El espíritu entre bastidores esperaba su hora. Por fin aparece. Se presenta. El espíritu en persona, el espíritu en espíritu y con todas las letras, el Geist se afirma sin comillas. Se afirma a través de la afirmación de la universidad alemana" (Derrida, 50).

El recorrido apuntado apenas en Ser y tiempo muestra de esta forma su inevitable final. Estaba claro, a pesar del lenguaje elusivo empleado, que los riesgos 
asumidos podían conducirnos a recalar en esta conocida ensenada. En ese contexto, como nos recuerda Derrida, Heidegger propone una definición de espíritu mediante el cuestionamiento (Fragen) de "la voluntad de saber y la voluntad de esencia", a través del "mundo, tema central de Ser y tiempo. Como una instancia del Fragen, marca la continuidad profunda que hay entre Ser y tiempo y el Discurso", por medio de los temas de la tierra y la raza, y por último, "todavía en continuidad esencial e interior con Ser y tiempo, la Entschlossenheit: la resolución" (Derrida, 55).

Los trazos pueden seguirse con claridad, siempre que seamos respetuosos con la verdad y no nos empeñemos, como se ha hecho tantas veces, en salvar a este pensador de sus propios demonios. Luz y oscuridad forman parte de esta filosofía, grande como pocas a pesar de la presencia en ella de algunos movimientos que desplazan contenidos que vienen cargados de ponzoña.

En el Discurso del Rectorado, cuando ya el peor de los venenos ha hecho su efecto, Heidegger señala que "solo un mundo espiritual garantiza al pueblo la grandeza; pues obliga a que la permanente decisión entre la voluntad de grandeza y el dejarse llevar a la decadencia, sea la ley que rige la marcha que nuestro pueblo ha emprendido hacia su historia futura" (Heidegger, La autoafirmación, 13).

Convengamos, pese a advertir hacia donde nos lleva este discurso -0 , como sugeríamos al principio de estas consideraciones, precisamente por saber a dónde nos lleva-, en que es la determinación de la naturaleza infecta de ciertos conceptos, lo que puede ayudarnos a recuperar la validez del resto (Derrida, 55 y ss.). Esta última es la tarea que nos ayuda, en conclusión, a hacernos con una filosofía por medio de la cual comprendemos que el uno teme elegir porque no quiere elegirse. En efecto, por medio de ella comprendemos que, quien se siente unido a la masa, no quiere sufrir la experiencia de la elección de una identidad diferenciada. La construcción, para el que no quiere elegir, es siempre reconstrucción de lo acaecido. Esa repetición, no obstante, no es un esforzado mantener en pie lo sucedido. Por el contrario, es un apoyarse en ello aunque se encuentre en ruinas.

\section{RELATAR LO ACAECIDO}

La manera en que el Dasein puede abordar la tarea de relatar lo acaecido viene determinada por la necesidad de superar los condicionamientos que le impone la forma de vivir el presente, ya que la absorción en la ocupación "en el 'simple vivir' de la ocupación cotidiana, el Dasein no se comprende jamás como si corriera a lo largo de una serie continua de puros 'ahora'. En virtud de este encubrimiento, el tiempo que el Dasein se da, tiene, por así decirlo agujeros" (Heidegger, Ser y tiempo, 424). De ahí que según Heidegger, resulte tan difícil reconstruir mentalmente lo que se ha hecho durante un lapso temporal concreto.

Se hace así hincapié en que la densidad del tiempo vivido es muy variable. En verdad sabemos que momentos plenos de un existir comprometido se incardinan en el interior de una generalidad de la existencia que hace del tiempo que la 
comprende una superficie esponjosa. Cuando se pretende remontar esa dificultad, es inevitable que aparezca la tentación de llenar esos huecos con elementos tomados del imaginario colectivo. Pero estos últimos, en lugar de responder a la naturaleza real de lo acaecido, son muchas veces una forma de ocultamiento de la misma.

Por otro lado, la reiteración monótona genera un vacío en la memoria, que es paralelo a la falta de sustancia de la acción. Produce la dispersión en un hacer inconcluyente, que absorbe el tiempo sin dar ocasión a emplearlo en actividades resolutivas. De esta forma, quien simplemente se deja llevar por la existencia, tiene siempre la sensación de estar desbordado por el tiempo, de no tener tiempo para nada. Pero "así como el que existe en forma impropia pierde constantemente el tiempo y nunca 'tiene' tiempo, así también el carácter distintivo de la temporalidad de la existencia propia es que esta existencia, en su resolución, nunca pierde el tiempo, y que 'siempre tiene tiempo"' (Heidegger, Ser y tiempo, 424).

Perder el tiempo y no tener tiempo vienen a ser las dos caras de una misma moneda. El tiempo fluye carente de densidad cuando nuestro estar no se halla enraizado en él. Por el contrario, cuando nuestro hacer está anclado en el tiempo nos movemos con él. En esa situación no experimentamos el paso del tiempo como un viento que nos azota y nos arrastra; a diferencia de ello, el tiempo aparece como un fluido y nosotros nos movemos en él.

En este sentido, si se me permite el pequeño inciso, sorprende que fuera tomado tan poco en serio el contenido de los versos de Eurípides, "¿Quién sabe si vivir es morir / y morir es vivir?". Como es sabido, despreciando la apelación que esas palabras contienen a la constitución íntima de nuestro ser, Aristófanes los ridiculizó en Ranas y Platón tampoco parece estimarlos demasiado cuando los toma en consideración en su diálogo Gorgias (493). Aquí Sócrates viene a decirle a Calicles que una vida entregada al placer desenfrenado es igual que la muerte, mientras que la vida temperante es siempre preferible. Así pues, Sócrates cree que lo afirmado por Eurípides en esos versos sería cierto tan solo si lo referimos a aquellos que no saben llevar una vida de templanza. Tal vez podría decirse que la idea de un pensar la vida y un pensar la muerte, entendidos como partes de un mismo proceso, pese a ser vislumbrada en esos versos, parecía ofender el sentido común de los griegos.

Hay, en todo caso, dos formas claras de interpretar la cuestión. En la primera de ellas, la vida se convierte en un limbo en el cual es imposible un hacer personal y diferenciado. En la segunda posibilidad, la comprensión de la temporalidad nos permite dotar de sentido al hacer $\mathrm{y}$, en consecuencia, poder construir su historia. Desde esta última perspectiva, cuando Heidegger se refiere al movimiento aparente del Sol en torno a la Tierra, entendemos bien lo que pretende decirnos al afirmar que "su periódico transitar lo toma en cuenta el Dasein arrojado en el mundo, el Dasein que — temporizando - se da tiempo a sí mismo. En virtud de la 
interpretación datante del tiempo, bosquejada desde el estar arrojado en el Ahí, el acontecer del Dasein es un acontecer cotidiano" (Heidegger, Ser y tiempo, 427).

Se trata de un acontecer trivial y a la vez determinante. La vida del sujeto heideggeriano es el tiempo que de alguna manera está en sus manos, pero el tiempo no está a la mano como pueda estarlo un útil al que recurrimos para realizar una determinada operación. El tiempo ni es herramienta ni es pura potencialidad, tampoco es materia que permita la construcción de cualquier cosa. Pensando en estos términos, cabría decir que en todo caso sería la propia existencia del Dasein la que podría adquirir el valor de un instrumento cuyas partes vibran en el tiempo. En tal sentido el Dasein espacializa el tiempo, si puede hablarse de este modo. Por eso nos dice Heidegger que, "el tiempo del que en la temporalidad del Dasein nos ocupamos está siempre ligado, en cuanto a su databilidad a un lugar del Dasein" (432).

De cualquier forma, hablando de modo genérico, el tiempo es percibido como ligado a un acontecer y, en el acaecer, el suceso no es sin lugar. Tampoco el tiempo aparece como temporalidad mediada por el pensamiento si no hay una distancia que lo separe de lo que la sucesión de aconteceres es en mí, de tal forma que el que contempla un suceso percibe que este tiene una situación en el tiempo y es una marca en el tiempo, pero no contempla el decurso del tiempo en su propia existencia de forma directa, no constata de inmediato su temporalidad.

Sin embargo, el tiempo posee un aspecto subjetivo, según Heidegger, “"más subjetivo' que todo posible sujeto". Eso es así, porque hace posible el cuidado y "no es algo que está-ahí ni en el 'sujeto' ni en el 'objeto', no está 'dentro' ni está 'fuera', sino que 'es' 'anterior' a toda subjetividad y objetividad, porque representa la condición de posibilidad de esta 'anterioridad' misma" (434). En este sentido, los conceptos de objetivo y subjetivo han de ser replanteados a la luz de la interpretación del ser como presencia en el tiempo. El tiempo mundano puede pensarse como tiempo de un sujeto. También el tiempo de un sujeto podría alcanzar toda la objetividad que quepa ser mostrada a la hora de enunciar el acontecer mundano.

Esta visión se opone, como sabemos, a la interpretación más común de la temporalidad. Esto es algo que queda claro cuando advertimos el carácter desenfocado de lo que Heidegger llama "la interpretación vulgar del tiempo", que insiste en su infinitud, en lugar de hacerlo en su realidad concreta como sucesión de ahoras (438). Es decir, si consideramos infinita esa sucesión, el tiempo pierde su posible densidad como tiempo presente. Esta se diluye en una eternidad sin dirección ni sentido. Así el tiempo del Dasein no sería un construir en el tiempo ni siquiera un hacer tiempo, sino más bien un dejarse temporizar.

\section{APROPIARSE EL TIEMPO O SER APROPIADOS POR/PARA ÉL}

Heidegger devela el secreto de una temporalización basada en el modo de existencia indiferenciada que podemos representar por medio de la idea de la inmersión en lo uno. En ella se pone de manifiesto que la conciencia de la 
mortalidad puede conducir a percibir la dimensión radicalmente subjetiva del tiempo (Heidegger, Ser y tiempo, 438). Frente a esta forma de apropiarse el tiempo, hay otras muchas apropiaciones que tienen un carácter ilusorio.

Estas son un tener tiempo que se traduce en un dejar de hacer, un tener tiempo para apartarnos del cumplimiento, para realizar las cosas más tarde. La equívoca confianza en que siempre podrán ser retomadas las acciones pendientes es una forma más del engaño que Heidegger ha denominado esquivamiento. A propósito de este punto, habría que subrayar - tal como hace el autor de Ser y tiempo - que no hay forma de esquivar la ruptura en la continuidad del tiempo que supone la muerte y se pregunta: “¿Por qué decimos: el tiempo pasa, y no decimos, con igual énfasis: el tiempo surge? Considerando la pura secuencia de los ahora, ambas cosas podrían decirse con igual razón" (439).

Es bien cierto que el tiempo surge y que emerge con él la posibilidad de existir. El tiempo surge porque lo hace en la posibilidad de ser. Al expresarme de esta forma no quiero confundir, aunque no siempre sea viable separar. Pero el tiempo discurre en esas y otras direcciones, siguiendo la sencilla línea de la continuidad, pero dando ocasión también a la quebrada línea de la indeterminación. No hay, pues, una única línea que vaya del pasado al presente y de este al futuro, existen muchas y son tortuosas. Se bifurcan una y mil veces, su capilaridad es tan intensa que no es posible trazar un mapa completo partiendo de las mismas (440).

Hay un acomodo del tiempo particular en el tiempo universal, viene a decirnos Heidegger. El tiempo particular no es reversible porque el tiempo universal tampoco lo es; no solo porque físicamente no lo sea, sino también por el peso que en la temporalidad del ser-ahí tiene la acción colectiva. No obstante, la idea de un tiempo del Dasein queda transfigurada después de ese acoplamiento. Por otro lado, si le concedemos alguna virtualidad, la tesis del eterno retorno nos llama a admitir en cierta forma la posibilidad de una reversibilidad del tiempo para el Dasein. No nos referimos con ello simplemente a la interpretación circular del tiempo, sino a la base, como ya se ha apuntado, que Friedrich Nietzsche estableció para su ética sin código. Esta viene a decirnos que hemos de actuar siempre de tal modo que no nos importe que la acción que acometemos se reitere de forma indefinida. Esa manera de temporizar no sería, como tantas otras, un pobre intento fallido de huir de la muerte. Antes al contrario, si nos fijamos en el ideal regulativo para la conducta que de ella puede derivarse, conduciría a una actitud más consecuente frente al hecho ineludible de la finitud de la existencia.

\section{LA TAREA FILOSÓFICA}

En cualquier caso, esa interpretación del tiempo forma parte de una cosmovisión que en estos momentos solo es recuperable como pasado. La tarea de integrarla en el presente y la labor de construir de antemano la cosmovisión en la que los elementos imprescindibles de aquella puedan integrarse, constituyen la 
faena filosófica por excelencia. Como bien sabemos, para Heidegger, "la filosofía es una ontología fenomenológica universal que tiene su punto de partida en la hermenéutica del Dasein, la cual, como analítica de la existencia, ha fijado el término del hilo conductor de todo cuestionamiento filosófico en el punto donde este surge y en el que, a su vez, repercute" (450).

Por ello, interpretar qué es el Dasein es la tarea primera de la filosofía (Fritsche, 266). De su éxito depende toda ulterior empresa teórica. Saber lo que, en última instancia, somos, delimitar el valor de la interpretación que habla en cada caso de la posibilidad de un llegar a ser lo que se es; estos son los cometidos primarios de la reflexión filosófica, al menos en la acepción consagrada por la tradición socrática.

Hemos visto cómo la pregunta por el ser no es fruto de la espontaneidad de la llamada conciencia natural, aunque tampoco en la racionalidad científica pueda encontrar los elementos adecuados para su formulación. Sabemos, en todo caso, que la ciencia puede contribuir a la respuesta, pese a que no sea capaz de aportar nada a la enunciación de la pregunta, aunque no ayude a comprender lo que el hecho mismo de esa enunciación implica. Heidegger añade que ni el origen ni la posibilidad de la idea del ser pueden ser investigados con los recursos de la lógica formal. Para él, "es necesario buscar un camino para la aclaración de la pregunta ontológica fundamental, y recorrerlo. Si es el único o el correcto, solo puede zanjarse después de la marcha. El debate acerca de la interpretación del ser no puede ser dirimido, porque ni siquiera se ha desencadenado" (Heidegger, Ser y tiempo, 450).

\section{A MODO DE CONCLUSIÓN}

Haciendo balance de la secuencia discursiva desplegada en Ser y tiempo hasta ese punto, señala asimismo que esa es la meta a la que se encamina. Entonces, al preguntarse por el momento en que dicha indagación se encuentra, acaba reseñando que "eso que llamamos el 'ser' está abierto en la comprensión del ser que, en cuanto comprender, es constitutiva del Dasein existente" (Heidegger, Ser y tiempo, 450). Y se refiere a una apertura preconceptual que otorgaría un primer impulso a esas ambiciones.

Heidegger termina así su obra fundamental reiterando algo anotado en los primeros compases de la misma, a saber: que la apertura del ser hacia la comprensión es la condición ineludible para entender el mundo y comprendernos a nosotros mismos. En ambos extremos habría una realidad subyacente que siempre ha escapado y que sigue escapando a la comprensión, a la que podríamos aludir recurriendo metafóricamente a la existencia de una proximidad esencial del ser y el pensar que explicaría la posibilidad de racionalización. Él no ofrece más detalles, pero analizando su propuesta y cotejándola con la de otros pensadores, en 
particular, como hacíamos al iniciar estas páginas, con la de Hegel, llegamos a colegir la existencia de un núcleo racional que ha de desplegarse en el tiempo, pero intuimos que, a diferencia de lo que planteaba Hegel, habrá de hacerlo siguiendo para ello un decurso específico y no respondiendo a una pauta fija de carácter universal. A este respecto, Sacristán recordaba de forma certera que Heidegger había hecho mención a que "él no busca, como Hegel, superación (Aufhebung), sino que practica un paso atrás, Schritt zurück. Cierto que el paso atrás no lleva a Heidegger hacia algo ya dicho por la tradición, sino precisamente a lo no pensado en ella, en el sentido de necesariamente implicado por lo pensado en ella" (Sacristán, 247).

El desvelamiento del tiempo originario, el significado del tiempo en cuanto tal y no en tanto que tiempo de algo o tiempo para algo, parecía prometer la revelación de los límites del ser. En cierto modo, la tarea acometida y desarrollada en Ser y tiempo ha cumplido, hasta el límite de lo posible, esa expectativa. Pero lo ha hecho abriendo la posibilidad de una precomprensión antes que proporcionando un conocimiento concreto. "¿Se revela el tiempo mismo como el horizonte del ser?" (Heidegger, Ser y tiempo, 451). Estas son las palabras finales de Ser y tiempo, un libro que Heidegger quiso que acabara igual que había empezado, con una apelación al enigma esencial del ser (Leyte, 14).

$\mathrm{Si}$ alguna apreciación podemos añadir es, ante todo, esta: el tiempo no es solo horizonte, es sustancia del ser. Aunque tal vez sería honesto añadir también alguna apostilla acerca de la certera experiencia, fenomenológicamente expuesta en la obra, de la dificultad de reconocerse tiempo. De algún modo, la superficie manifiesta el interior, de la misma manera que la forma muestra el fondo, pero esto no es suficiente. Heráclito nos enseñó a amar el fuego en tanto que metáfora del devenir y aceptar la discordia como principio rector de cuanto es. Nos dijo que el tiempo es como un niño jugando a entorpecer los movimientos de otro niño. El tiempo es un niño porque siempre es nuevo, porque no retorna a sí mismo, sino a condición de retornar a otro, porque, como ya se ha sugerido, aun rebosando de sangre y vergüenza no puede perder del todo la inocencia.

Cada uno de nosotros quiere jugar con el tiempo y, por ello entorpece como puede sus movimientos para que él no pueda entorpecer los nuestros. Pero nosotros volvemos cansados a casa, nos cruzamos con otros cada noche, con frecuencia hablamos con alguien que está más anulado cada vez, aunque no sepa por qué, alguien que tal vez no pueda decirnos de dónde le viene esa fatiga indefinida que le hace encontrarse a sí mismo entre los pliegues del tejido con el que está hecho el vestido del que al acabar el día se despoja.

El tiempo, por su parte, es de nuevo niño cuando al alba la luz nos viene al encuentro. El tiempo es un niño sabio, que conoce por donde asomarse al vacío, así como el modo de ver lo que hay dentro y fuera del juego al que está jugando. Claro que, con esta manera heideggeriana de representar el tiempo, la única en que 
conseguimos visualizar lo que queremos expresar, lo devolvemos al plano de lo metafórico, alejándonos de esta forma por última vez de nuestro intento de aproximación al orden de los conceptos.

Universidad de La Laguna*

Facultad de Humanidades. Sección Filosofia

Campus de Guajara s/n (38071), La Laguna. Tenerife (España)

dferagi@ull.edu.es

\section{OBRAS CITADAS}

Babich, Babette. “'A Philosophical Shock': Foucault's Reading of Heidegger and Nietzsche". En: Prado, C. G. (ed). Foucault's Legacy. London: Continuum, 2009.

Deleuze, Gilles. Foucault. Barcelona: Paidós, 1987.

Derrida, Jacques. De l'esprit. Heidegger et la question. Paris: Galilée, 1987.

Di Giovanni, Piero. "Etica, politica e metafisica". En VVAA. Heidegger e la filosofia practica, Palermo: S. F. Flaccovio Editore, 1994.

Foucault, Michel. "Soggettività e verità". En: Foucault, M. Sull'origine dell'ermeneutica del sé. Due conferenze al Darmouth College. Napoli: Cronopio, 2012.

Mal faire, dire vrai. Louvain: Presses universitaires de Louvain, 2012.

Fritsche, Johannes. "Heidegger's Being and Time and National Socialism". Philosophy Today 56/3 (2012).

Gadamer, Hans Georg. Verdad y método. Vol. I. Salamanca: Sígueme, 1987.

Heidegger, Martin. Ser y tiempo. Madrid: Trotta, 2005. La autoafirmación de la universidad alemana. Madrid: Tecnos, 1989.

Krockow, Christian Graf. La decisión. Un estudio sobre Ernst Jünger, Carl Schmitt y Martin Heidegger. México: CEPCOM, 2001.

Leyte, Arturo. Heidegger. Madrid: Alianza, 2005.

Platón. “Gorgias”. En: Platón. Diálogos. Vol. II. Madrid: Gredos, 1983.

Plutarco. Anziani e política. Palermo: Sellerio editore, 1991.

Quesada, Julio. "Heidegger: de la tarea hermenéutica como 'destrucción' a la 'selección racial' como metafísicamente necesaria, pasando por la adhesión al nacionalsocialismo. La experiencia de Ser y tiempo". Contrastes XVII (2012).

Sabot, Philippe. "Linguaio, società, corpo". Materiali Foucaultiani I/1 (2012). 
Sacristán, Manuel. Las ideas gnoseológicas de Heidegger. Barcelona: Crítica, 1995.

Tirvaudey, Robert. "L'ipséité et l'altérité en question: Heidegger, Sartre, Kierkegaard". Revue philosophique de la France et de l'étranger 137/3 (2013). 